\title{
Analytical Solution of an Irreversible Surface Reaction Model
}

\author{
E. Clément, ${ }^{1}$ P. Leroux-Hugon, ${ }^{2}$ and L. M. Sander ${ }^{2,3}$
}

\begin{abstract}
In this work, we consider a simple model of reaction-limited annihilation $A+B \rightarrow 0$ with a random source and desorption in the spirit of the reaction model proposed by Fitchthorn, Ziff, and Gulari, and we solve it exactly using a spin model. We show that the situation is similar to a diffusion-limited kinetic situation (though diffusion was a priori absent from the model). We find the occurrence of a large-scale organization phenomenon at low dimension called segregation, and for a finite size system, a transition to a saturated state at low desorption probability. We show how this transition is affected by the dimensionality of the substrate. We also show how the fluctuation spectrum of two quantities such as the saturation and the reaction rate can be drastically different, the first being universal and the other sensitive to the geometry of the substrate.
\end{abstract}

KEY WORDS: Reaction kinetics; catalysis; spin model.

\section{INTRODUCTION}

Heterogeneous catalysis is a field that exhibits a large choice of reactive processes. These processes are of central importance for numerous industrial and practical applications. Moreover, this field contains a great wealth of still unclear or completely unexplained phenomena which creates a challenging domain of investigation for fundamental research. Even the most basic heterogenous bimolecular reaction, e.g., the conversion of carbon monoxyde on platinium, shows empirically a great deal of

\footnotetext{
${ }^{1}$ Laboratoire d'Acoustique et d'Optique de la Matière Condensée, Université Paris-VI, 75005 Paris, France.

${ }^{2}$ Groupe de Physique du Solide, Université Paris-VI and Paris-VII, 75005 Paris, France.

${ }^{3}$ Department of Physics, University of Michigan, Ann Arbor, Michigan 48104.
} 
unexpected and unclear behavior. For example, it has been shown experimentally that under appropriate conditions, the rate of oxidation on a Pt surface exhibits sustained temporal oscillations which are associated with an adsorbate-induced surface transformation. ${ }^{(3)}$ Recently, several models have been proposed ${ }^{(1,3)}$ to unravel these mysterious behaviors; they mainly emphasized the irreversible dynamical behavior of a bimolecular system, beyond the scope of classical statistical mechanics. We consider here a model that was first proposed by Fichthorn, Ziff, and Gulari $(\mathrm{FZG}){ }^{(1)}$

The FZG model consists of three steps:

1. A bimolecular reaction: $A+B \rightarrow x x$ (two vacant sites).

2. A symmetric desorption: $\mathrm{A} \rightarrow \mathrm{x}$ and $\mathrm{B} \rightarrow \mathrm{x}$ (with the same probability $p$ ).

\section{A symmetric adsorption: $\mathrm{x} \rightarrow \mathrm{A}$ or $\mathrm{B}$.}

The fast step is adsorption: at the time scale on which the process is analyzed, the sites are always filled with A or with B (no empty site). This model is so simple that it may not represent any actual reactive process. Nevertheles, it is a very interesting one because it allows us to extract important conceptual information that can be extremely useful in the field of reaction kinetics. We show in particular how the confiment of reactants to a medium with a dimension smaller than two requires one to consider a solution that goes beyond a mean-field treatment, which means that the implicit assumption of a uniform distribution of reactants on the surface is wrong. This model was first numerically studied by $\mathrm{FGZ}^{(1)}$ and later a mean-field solution was proposed by Considine et al. ${ }^{(4)}$ Both predicted the occurrence of a bistability transition at a low desorption probability. We choose here to reconsider the model because we found that an interesting self-organization phenomenon is present. In a second step, we analyze both theoretically and through computer simulations the fluctuation spectrum of two macroscopic parameters: the saturation and the reaction rate.

\section{THE MASTER EQUATION}

We map the system onto a dynamic spin model where a spin up represents one species (say A) and a spin down represents another species. A bimolecular reaction is always followed by adsorption, and a desorption is followed by an immediate adsorption. This can be decomposed into a set of elementary spin interactions. A master equation is then written that contains all the dynamical information on the system. We establish here the equation of motion for the first two moments of this equation. The interest 
of the model lies in the fact that both sets of equations are in closed form. The lattice is taken to be a $d$-dimensional Euclidean space and whenever necessary a hypercubic lattice with cyclic boundary conditions. The linear size is $L$ and the total number of sites is $N=L^{d}$. We define the spin parameter to be $z_{j}(+1$ or -1$)$. The reaction and adsorption are decomposed into four processes. Consider one spin $z_{j}$ and the interaction with a neighbor $z_{j+x}$. If and only if these spins are opposed we have simultaneously the flipping process $\left(z_{j}, z_{j+\alpha} \rightarrow-z_{j}, z_{j+\alpha}\right)$, the exchange process $\left(z_{j}, z_{j+\alpha} \rightarrow-z_{j},-z_{j+\alpha}\right)$, the transfer process $\left(z_{j}, z_{j+\alpha} \rightarrow z_{j}\right.$, $-z_{j+\alpha}$ ), and an identity process, each one with the same probability $1 / 4$. The jump probabilities are, respectively, $W F_{j, \alpha}=W E_{j, \alpha}=W T_{j, \alpha}=$ $1 / 16 d\left(1-z_{j} z_{j+x}\right)$. For desorption, the jump probability is $W D_{j, x}=1 / 2 p$. We write a master equation for the probability $P(\{z\})$ to observe the spin configuration $\{z\}$ :

$$
\begin{aligned}
\dot{P}(\{z\})= & \sum_{j} \sum_{\alpha}\left[W F_{j, \alpha}\left(\left\{-z_{j}\right\}\right) P\left(\left\{-z_{j}\right\}\right)+W F_{j, \alpha}(\{z\}) P(\{z\})\right. \\
& +W E_{j, \alpha}\left(\left\{-z_{j},-z_{j+\alpha}\right\}\right) P\left(\left\{-z_{j},-z_{j+\alpha}\right\}\right)-W E_{j, \alpha}(\{z\}) P(\{z\}) \\
& \left.+W T_{j, \alpha}\left(\left\{-z_{j+\alpha}\right\}\right) P\left(\left\{-z_{j+\alpha}\right\}\right)-W T_{j, \alpha}(\{z\}) P(\{z\})\right] \\
& +\sum_{j} W D_{j}\left(\left\{-z_{j}\right\}\right) P\left(\left\{-z_{j}\right\}\right)-W D_{j}(\{z\}) P(\{z\})
\end{aligned}
$$

This equation describes a process slightly different than the FZG model, but nevertheless both models are identical when $p \ll 1$. Solving for the first moment $\gamma_{j}=1 / N \sum_{j} \sum_{\{z\}} z_{j} P\{z\}$ leads to the equation

$$
\dot{\gamma}=D \Delta_{j} \hat{\gamma}_{j}-p \gamma_{j}
$$

with the notations

$$
\Delta f_{j}=\sum_{\alpha}\left(f_{j+\alpha}-f_{j}\right)
$$

and

$$
D=1 / 2 d
$$

We recognize an effective diffusion term (with a diffusion constant $D$ ) and a desorption term. The diffusion term is a surprise, since no diffusion was put into the model. This is a consequence of the nearest neighbor reaction term in association with the fast absorption process. This equation is identical to the one we derived for a diffusion-limited annihilation process. ${ }^{(4)}$ Unfortunately, the solution of this equation contains only trivial 
information: the steady-state solution is identically zero, which just means that for each realization there is a symmetric one obtained by inverting all the spins and which has the same probability at steady state. In order to investigate the structural properties of the model, we have to go to a higher moment. We established the equation of motion for $m_{k}=1 / N \sum\left\langle\gamma_{j} \gamma_{j+k}\right\rangle$; $m_{k}$ is a correlator that tells us up to which distance two spins are likely to be in the same direction. We obtain

$$
\dot{m}_{k}=2 D \Delta_{k} m_{k}-2 p m_{k}-2 D Q \sum_{\alpha} \delta_{x, k}+2(2 Q+p) \delta_{0, k}
$$

The notation $\delta_{\alpha, k}$ is a Kronecker symbol for the vectorial indices $\alpha$ and $k$. We can easilly interpret the physical origin of all the terms of this equation. It contains an effective diffusion term $\left(\Delta_{k} m_{k}\right)$, a contact reaction term with a nonzero value for $k=\alpha$, and a desorption term. The term which is nonzero for $k=0$ is the one that keeps $m_{0}=1$ at all times and besides this term, this equation is reminiscent of an equation we have already found in the diffusion-limited case with desorption. The parameter $Q$ is the probability to have two opposite spins nearest neighbor in a given direction $\alpha$ (since the model is isotropic, $Q$ is the same in every direction). $Q$ can be interpreted as a reaction probability. This set of coupled equations, though simple, contains nontrivial features. We propose here to investigate (1) more deeply, since we are now aware that it not only solves the FGZ model, but also gives important information on the diffusion-limited problem. It is worth noticing that Eq. (1) is in a closed form, which is a direct consequence of the transition probability containing only quadratic terms and that the spin algebra has the property $z_{i}^{2}=+1$. In a same manner it is easy to see that all the moments of $z_{j}$ decouple. In this paper we concentrate on steady-state properties. The dynamical behavior (approach to steady state) also shows some interesting features and will be discussed elsewhere. ${ }^{(5)}$

\section{THE STEADY STATE}

Now we are interested in the steady-state properties of the FGZ model when the desorption probability goes to zero. The investigate the transition toward a saturated state, we define an order parameter

$$
\Delta_{\mathrm{AB}}=\left\langle\left(N_{\mathrm{A}}-N_{\mathrm{B}}\right)^{2}\right\rangle / N^{2}
$$

$N_{\mathrm{A}}$ and $N_{\mathrm{B}}$ are the numbers of $\mathrm{A}$ and $\mathrm{B}$ on the lattice. This parameter is 
unity when the system is totally saturated. We define the spatial Fourier transform

$$
\begin{aligned}
& \ddot{f}_{q}=\sum_{j} f_{j} e^{i 2 \pi / L q \cdot j} \\
& f_{r}=\sum_{q} \bar{f}_{q} e^{-i 2 \pi / L q \cdot j}
\end{aligned}
$$

It is easy to check that $\Delta_{\mathrm{AB}}$ is the zero mode of the Fourier coefficients of $m_{k}$. A Fourier analysis of Eq. (1) at steady state gives for the $q$ mode

$$
\bar{m}_{q}=\frac{1}{L^{d}}\left(Q+\frac{Q(1-p)+p}{2 D \sum_{j=1}^{d}\left(1-\cos 2 \pi q_{j} / L\right)+p}\right)
$$

Therefore, we have

$$
\Delta_{\mathrm{AB}}=1 / N(1+Q / p)
$$

Equation (2) shows how the reaction rate and the saturation parameter are closely related in the steady-state limit.

The correlation function $m_{k}$ yields

$$
m_{k}=Q \delta_{k, 0}+\frac{Q(1-p)+p}{L^{d}} \sum_{q} \frac{\cos \left(2 \pi / L \sum_{j=1}^{d} k_{j} q_{j}\right)}{2 D \sum_{j=1}^{d}\left(1-\cos 2 \pi q_{j} / L\right)+p}
$$

Thus we calculate $Q$ and obtain

$$
Q=1 / 2 \frac{1-p F_{d}(L, p)}{1+\frac{1}{2}(1-p) F_{d}(L, p)}
$$

with

$$
F_{d}(L, p)=\frac{1}{L^{d}} \sum_{q} \frac{\cos \left(2 \pi / L \sum_{j=1}^{d} k_{j} q_{j}\right)}{2 D \sum_{j=1}^{d}\left(1-\cos 2 \pi q_{j} / L\right)+p}
$$

This function $F_{d}(p, L)$ has a central importance in this theoretical investigation, since its scaling behavior as a function of $p, L$, and the dimension $d$ answers the questions we address here. First, let us notice that Eq. (2) shows that in the thermodynamic limit, no saturation is possible $\left(\Delta_{\mathrm{AB}}=0\right.$ when $\left.N \rightarrow \infty\right)$. For a finite size system, a saturation will occur if $Q / p \cong N$. Furthermore, careful analysis of (4b) shows that the results can be interpreted as a function of a reduced variable $X$ which depends on $p$, 
$L$, and the dimension $d$, and that determines the situation toward the saturation transition. We find

$$
\begin{aligned}
& X=L p^{1 / 2} \quad \text { for } \quad d=1 \\
& X=L^{2} p \ln 1 / p \quad \text { for } \quad d=2 \\
& X=L^{3} p \quad \text { for } \quad d=3
\end{aligned}
$$

At very low desorption probability $p$, i.e., for $X \ll 1$, the leading term in Eq. (4b) is found to be in any dimension $1 / p L^{d}$. The consequence is that we have a saturated state and we obtain

$$
\begin{aligned}
\Delta_{\mathrm{AB}} & \cong 1 \\
Q & =L^{d} p
\end{aligned}
$$

Now, let us consider intermediate values of the desorption parameter, i.e., $X \gg 1$; we approximate the discrete sum (4b) over the modes $q_{j}$ by an integral where the $L$ dependence has disappeared:

$$
F_{d}(L, p) \rightarrow I_{d}(L, p)=\int_{0}^{p / 2} d x_{1} \cdots \int_{0}^{\pi / 2} d x_{d} \frac{\cos \left(x_{1}\right)}{2 D \sum_{j=1}^{d}\left(1-\cos x_{j}\right)+p}
$$

This regime is similar to the thermodynamic limit investigated in ref. 6 . The system is sensible to low-dimensional confinement and in particular, for dimensions $d \leqslant 2$, we obtain a mesoscopic segregation corresponding to the occurrence of domains of size $1 \ll A \ll L$. The self-organization scale $A$ of the system can be estimated with the help of the correlation function $m_{k}$. It is defined to be the scale for which $m_{k}$ shows no correlation and reaches the value $\bar{m}_{0}=\Delta_{\mathrm{AB}}$. Since the regime $X \gg 1$ defines a limiting situation where the saturation has no macroscopic relevance (i.e., $\bar{m}_{0}=\Delta_{\mathrm{AB}} \cong 0$ ) and the reaction rate $Q \ll 1$ (i.e., $m_{x} \cong 1$ ), we define the saturation length $A$ such that

$$
-\Lambda \alpha \cdot \nabla m_{\alpha} \equiv 1
$$

In $d=1$, we obtain in the limit $p \ll 1$, after calculation of the Eq. (3) when the sum is replaced by an integral,

$$
m_{k}=\exp \left[-k\left(\frac{p}{D}\right)^{1 / 2}\right]
$$

The segregation size is

$$
A \cong(D / p)^{1 / 2}
$$


and we have a scaling behavior for the reaction rate and the saturation parameter

$$
\begin{aligned}
Q & =(p / D)^{1 / 2} \\
\Delta_{\mathrm{AB}} & =(p D)^{-1 / 2} L^{-1}
\end{aligned}
$$

In $d=2$ we extract the scaling behaviors

$$
\begin{aligned}
A & \cong \ln 1 / p \\
Q & =2 \pi D(\log 1 / p)^{-1} \\
\Delta_{\mathrm{AB}} & =2 \pi D /\left(p \log 1 / p L^{2}\right)
\end{aligned}
$$

This dimension $d=2$ is the marginal dimension of the problem. In $d=3$, we obtain

$$
\begin{aligned}
A & \cong 1 \quad \text { (microscopic size) } \\
Q & =1 / 2 \\
\Delta_{\mathrm{AB}} & =1 /\left(2 p L^{3}\right)
\end{aligned}
$$

In summary, we have a transition at low description probability toward a saturated state for $X<1$. The transition corresponds physically to a random fluctuation in the difference of A's and B's which is not triggered by a spatial organization, but generated by the source of particles. For a fixed number of sites, the lower the dimension, the lower is the transition occurring in $p$. The reaction rate is drastically smaller than what is predicted by the mean-field solution It is a direct consequence of the mesoscopic self-organization of the system at a scale $A$. We find therefore for $d \leqslant 2$

$$
\Delta \approx p^{d / 2-1}
$$

For $d=1$, a saturation is observed when the size of the system is comparable to the segregation length $A$ (i.e., when $X \cong 1$ ). The transition is shown in Figs. 1 and 2. If Fig. 1, we plot the saturation parameter $\Delta_{\mathrm{AB}}$ for a fixed total number of sites $N=4096$, in dimension $d=1, d=2$, and $d=3$, as a function of the desorption probability. In addition, we plot the result of the mean-field calculation by Considine et al. ${ }^{(4)}$ Clearly, for $d \leqslant 2$ the mean-field calculation breaks down; In Figs. $2 \mathrm{~A}$ and $2 \mathrm{~B}$, we represent the theoretical saturation parameter and the results of Monte Carlo simulations, as a function of the reduced variable $X$, for different dimensions, 


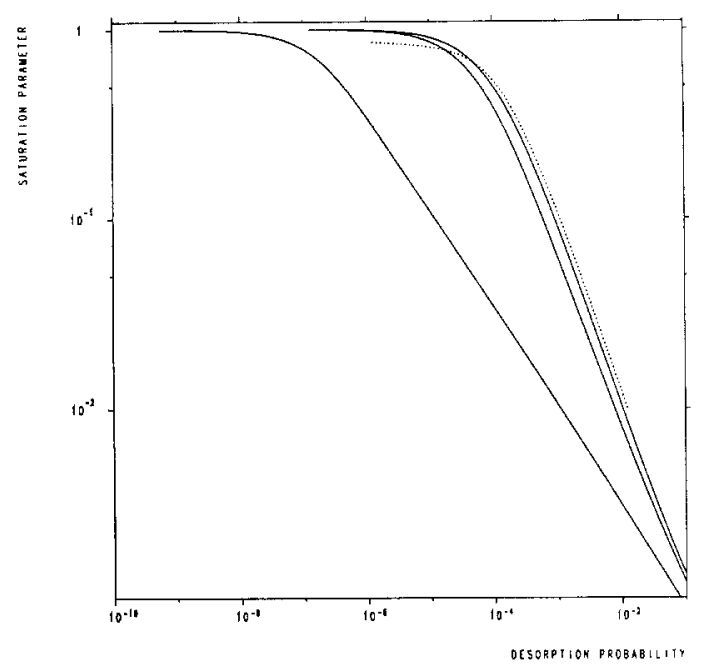

Fig. 1. $\Delta_{\mathrm{AB}}$ as a function of $p$. Solid lines: $d=1 \quad(N=4096), d=2(N=64 * 64)$, and $d=(16 * 16 * 16)$. Dotted line: mean-field result. ${ }^{(4)}$

system sizes, and desorption probabilities. The collapse of the data on a master curve is clear.

Note that in real catalysis problems, the case of $d=1$ is not totally academic, since a catalytic surface is rarely plane and bidimensional and it is known now that the reactants have a preferential reaction probability on the steps of the terraces. Along this line, another interesting problem is the generalization of this study to fractal structures The form of Eq. (1) suggests a result similar to the one found in the diffusion-limited problem. ${ }^{(7)}$ In the fractal case, with a spectral dimension $d_{s}$, we suggest, according to these previous results, that the behavior of the segregation scale and the saturation transition would be similar to the Euclidean case if one replaces in Eq. (5) the Euclidean dimension $d$ by the spectral dimension $d_{s}$. This mean a strong effect is expected for most geometrically disordered systems which have in general $d_{s} \leqslant 2$.

\section{FLUCTUATION SPECTRA}

Here we investigate the influence of the saturation and the segregation on the fluctuation spectrum of the total saturation $\Gamma(t)=1 / L^{d} \sum \gamma_{r}(t)$ and the reaction rate $Q(t)$. We are in particular interested in the role of the dimension and the effect of the finite size.

The total saturation $\Gamma(t)$ reveals the total excess in a given species; it is a quantity that fluctuates around $\langle\Gamma(t)\rangle=0$ and the standard deviation 


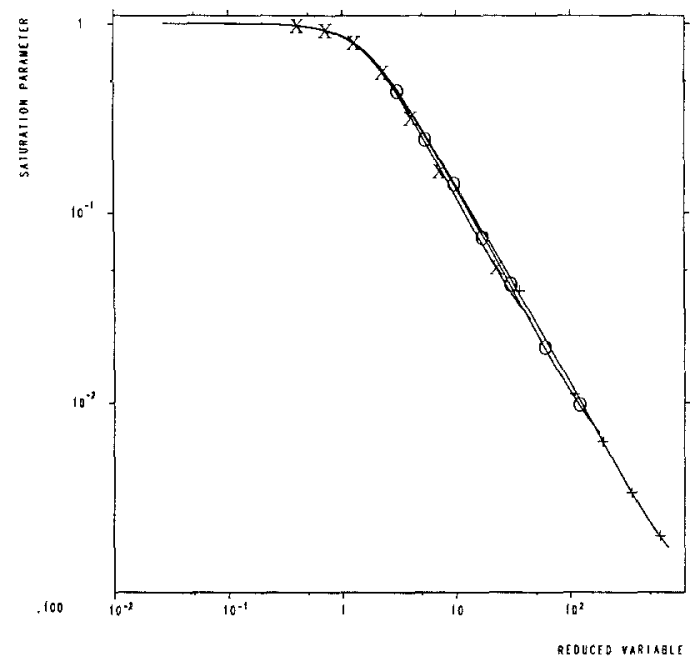

(A)

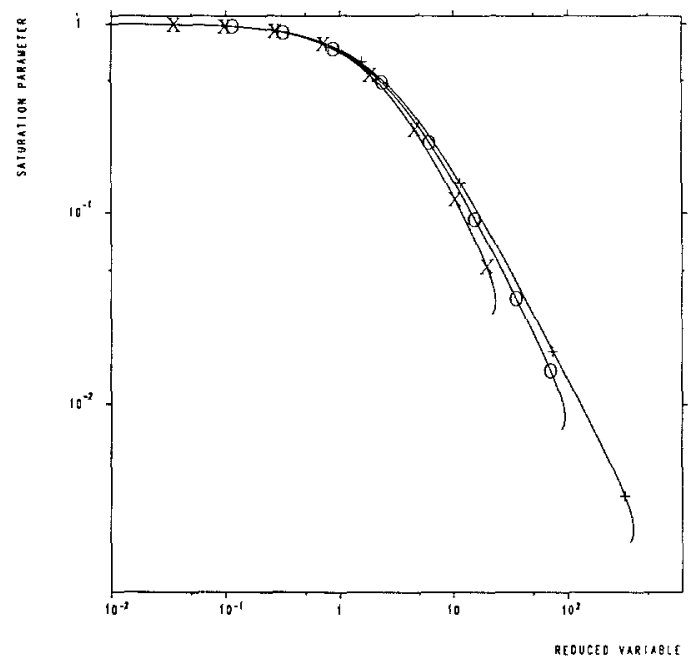

(B)

Fig. 2. $\Delta_{\mathrm{AB}}$ as a function of the reduced variable $X$. Solid lines are theoretical calcullations and symbols denote Monte Carlo simulations. (A) $d=1, X=N p^{1 / 2}, N=64(\times), N=256$ (O), and $N=1024(+)$. (B) $d=2, X=N p \log (1 / p), N=64(\times), N=256(O)$, and $N=1024(+)$. 
of which is the saturation parameter $\Delta_{\mathrm{AB}}$. To obtain the fluctuation spectrum, we design a Langevin approach already used in previous works. ${ }^{(6,8,9)}$ We write a Langevin equation for the first moment

$$
\dot{\gamma}_{r}(t)=D \Delta \gamma_{r}(t)-p \gamma_{r}(t)+\eta_{r}\left(t^{\prime}\right)
$$

$\gamma_{r}(t)$ is the value of the spin variable at time $t$ and at the site labeled by the vectorial index $r$. Here $\eta_{r}(t)$ is the "random force" associated with the microscopic fluctuations. We take

$$
\begin{aligned}
\left\langle\eta_{r}(t)\right\rangle & =0 \\
\left\langle\eta_{r}(t) \eta_{r}^{\prime}\left(t^{\prime}\right)\right\rangle & =B\left(r-r^{\prime}\right) \delta\left(t-t^{\prime}\right)
\end{aligned}
$$

The noise is local in time and its spatial structure is chosen to reproduce the steady-state result of the second moment given by Eq. (1). We solve Eq. (6), and we obtain

$$
\begin{aligned}
\gamma_{r}(t)= & \sum_{q} \bar{\gamma}_{q}(0) \exp \left(-i 2 \pi / L q \cdot r-K_{q} \cdot t\right) \\
& +\sum_{q} \int_{0}^{t} d t^{\prime} \exp \left[--K_{q} \cdot\left(t^{\prime}-t\right)-i 2 \pi / L q r\right] \cdot \bar{\eta}_{q}\left(t^{\prime}\right)
\end{aligned}
$$

with

$$
K_{q}=2 D \sum_{j=1}^{d}\left(1-\cos 2 \pi / L q_{j}\right)+p
$$

Identification of

$$
\frac{1}{L^{d}} \sum_{r}\left\langle\gamma_{r}(t) \gamma_{r+k}(t)\right\rangle
$$

calculated from (7) with Eq. (3) yields, in the limit $t \gg 1 / p$, the equivalence condition

$$
\bar{B}_{q}=\frac{1}{L^{d}}\left[2 Q K_{q}+2 Q(1-p)+p\right]
$$

where $Q$ is the steady-state reaction rate. Now we are in a position to calculate the fluctuation spectrum of $\Gamma(t)=1 / L^{d} \sum \gamma_{r}(t)$, which is given by the Fourier transform of the autocorrelation function $C(\tau)=$ $\langle\Gamma(t) \Gamma(t+\tau\rangle$. We obtain

$$
C(\tau)=\Delta_{\mathrm{AB}} \exp (-p \tau)
$$




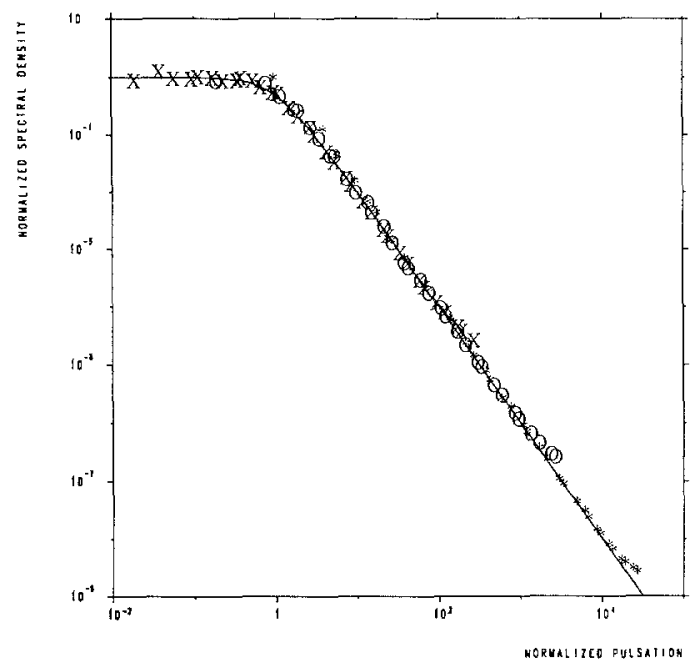

(A)

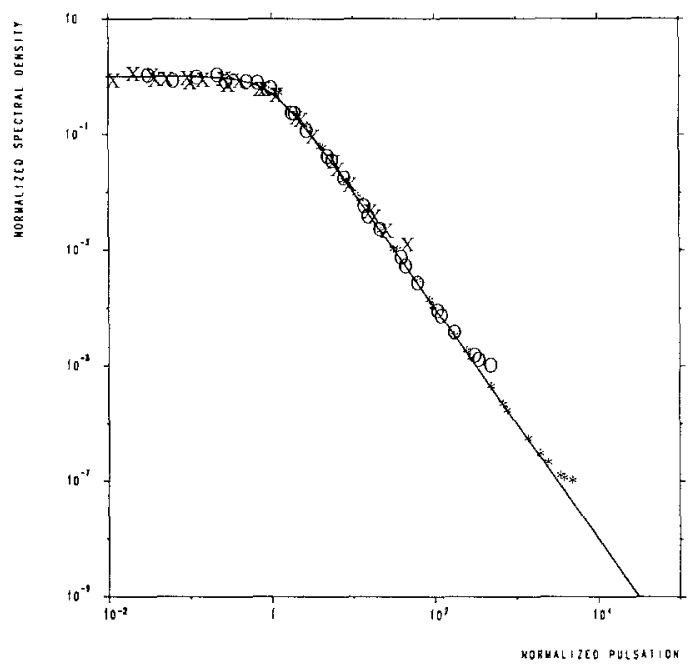

(B)

Fig. 3. Saturation parameter fluctuation $S(\omega)$ normalized by $\Delta_{\mathrm{AB}} / p$ as a function of $y=\omega / p$. (A) $d=1$; (B) $d=2$. Monte Carlo simulation (symbols) and theoretical expectation $\left(1+y^{2}\right)^{-1}$ (solid line). 
and a spectrum

$$
S^{\Delta}(\omega)=\Delta_{\mathrm{AB}} / p /\left(1+y^{2}\right) \quad \text { with } \quad y=\omega / p
$$

This spectrum corresponds to a simple exponential relaxation with a correlation time $1 / p$, and does not depend on the dimension of the substrate. We also note that an exponential decay with this specific correlation time was obtained by us with a mean-field calculation using a Van Kampen expansion ${ }^{(10)}$ of the Fokker-Planck equation derived by Considine et al. In Fig. 3 we represent the normalized spectrum $S^{\Delta}(\omega) p / \Delta_{\mathrm{AB}}$ as a function of $y$ for various conditions. The agreement with the theory is always excellent. Therefore, it is clear that the shape of the spectrum is universal, independent of the dimension, and coincides with a mean-field result (except of course for the prefactor value $\Delta_{\mathrm{AB}}$ ). Therefore, we conclude that a typical population change (which corresponds to a random inversion of the excess in A or in B) is achieved in times of the order of $1 / p$ and is only triggered by the fluctuations of the source, independent of the spatial organization of reactants.

Now we are interested in the reaction rate fluctuations. Fichthorn et al. ${ }^{(11)}$ have shown in $d=2$, on simulations, that these fluctuations offer an interesting dynamical behavior. When $p$ is decreased, the noise level is highly increased and sudden bursts of reaction are produced, followed by periods of dead calm. They also show that the noise is intimately related to the mean surface coverage in one species [equivalent to the saturation parameter $\Delta_{\mathrm{AB}}(t)$ in our language], which means that for a high coverage of one species the rate is low and the rate is maximum at equal coverage. We want to go a little bit further in this analysis and show that the reaction noise has a really complex behavior which is dependent not only on the saturation, but on the dimension and the self-organization of reactants. We performed Monte Carlo simulations in $d=1$ and $d=2$ for a finite size $N=100$ sites (see Fig. 4). The fluctuation spectrum behaves like a low-pass filter, but the frequency cutoff saturates to a fixed lower value as we come across the transition (i.e., $X \leqslant 1$ ). For $X<1$ the normalized spectrum seems to show a constant shape. The cutoff frequency varies with the size of the system (see Fig. 5) and the slope is $\alpha=-2$ in $d=2$ and $\alpha=-1.5$ in $d=1$. For $X>1$ the cutoff frequency shifts to higher values and the slope is increased. It is noticeable that in $d=1$ the slope reaches a values $\alpha$ close to -1 for large $X$. We performed a Langevin calculation on the reaction rate using a Gaussian decoupling of the noise at the level of the four times correlation function. The behavior we obtained could not reproduce the features we describe here, which means that it is necessary to go to the fourth moment calculation in order to study this surprising behavior. 


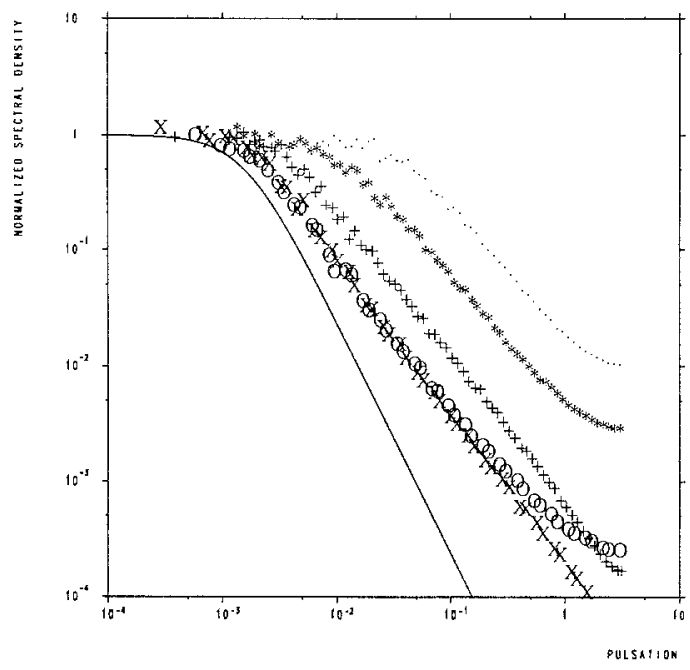

(a)

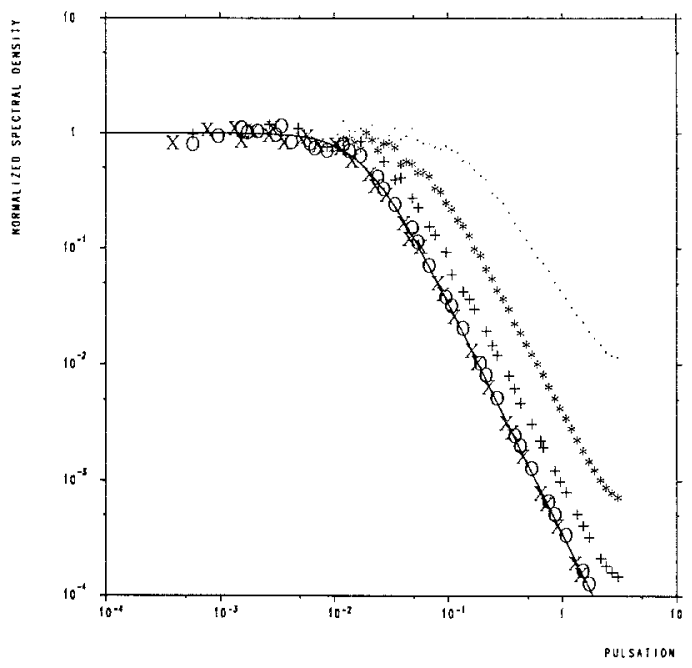

(b)

Fig. 4. Reaction fluctuation $R(\omega)$ normalized by $R(1)$ as a function of $\omega$. Monte Carlo simulations (symbols) and curve $\left(1+\omega^{2} / \omega_{c}^{2}\right)^{-1}$ (solid line). (a) $d=1$ for $N=100$ and $X=1.0$ ( $\times), X=1.78(0), X=3.16(+), X=5.62(*)$ and $X=10$. (.). (b) $d=2$ for $N=100$ and $X=0.06(\times), X=0.36(\bigcirc), X=3.17(+), X=7.76(*)$, and $X=17.3(\cdot)$. 


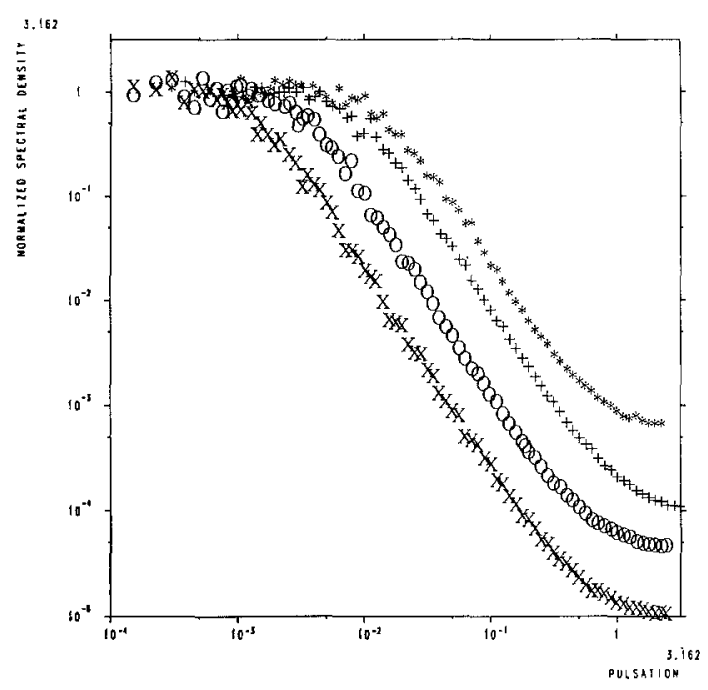

Fig. 5. Results of Monte Carlo simulation: spectrum of the reaction rate fluctuations $S_{60}$, normalized by the value at low frequencies after the transition $(X=0.3)$ for different system sizes in dimension $d=2:(*) L=7,(+) L=10,(\bigcirc) L=14,(\times) L=20$.

\section{CONCLUSIONS}

We have shown for a simple reaction-limited model with desorption that the presence of a high rate of adsorption could lead to a behavior recalling a diffusion-limited model. For an infinite system the effective diffusion term leads to a mesoscopic scale of organization controlled by the rate of desorption $p$. This effect disappears for $d>2$. For a finite size system, at low $p$, this self-organization is still present, but a transition to a saturated state occurs at lower $p$. The transition is monitored by the dimension of the space. At low dimension $(d \leqslant 2)$ the system is much more resistant to saturation and the reaction rate is lower than what is predicted by a meanfield theory. This study also shows, in analogy with diffusion-limited systems, that a self-organization scale is likely to persist for fractal substrata with a spectral dimension lower than two. We also show the drastic difference in the fluctuation spectra of the saturation (excess of one reactant) and the reaction rate, the first being universal and the other dependent on the dimension. This indicates the fundamentally different origin of the saturation and the segregation. The saturation is controlled by the fluctuation of the source and is independent of the segregation. On the other hand, the reaction rate depends strongly on the self-organization inside the system as well as the transition parameter. 


\section{ACKNOWLEDGMENTS}

L.M.S. is supported by NSF grant DM-88-15908. The OMC and the GPS are associated with the CNRS.

\section{REFERENCES}

1. K. Fichthorn, E. Gulari, and R. Ziff, Phys. Rev. Lett. 63:1527 (1989).

2. M. Cox, G. Ertl, and R. Imbihl, Phys. Rev. Lett. 54:1725 (1985).

3. R. Ziff, E. Gulari, and Y. Barshad, Phys. Rev. Lett. 56:2553 (1986).

4. D. Considine, S. Redner, and H. Takayasu, Phys. Rev. Lett. 63:2857 (1989).

5. C. Flament, E. Clément, P. Leroux-Hugon, and L. Sander, in preparation.

6. E. Clément, L. Sander, and R. Kopelman, Phys. Rev. A 39:6455 (1989); Phys. Rev. A 39:6466 (1989).

7. E. Clément, L. Sander, and R. Kopelman, Chem. Phys. 146:343 (1990).

8. Z. Y. Zhang, Phys. Rev. Lett. 59:1726 (1989).

9. K. Lindenberg, B. West, and R. Kopelman, Phys. Rev. Lett. 60:1777 (1989).

10. N. G. Van Kampen, Stochastic Processes in Physics and Chemistry (North-Holland (North-Holland, Amsterdam, 1984).

11. K. Fichthorn, E. Gulari, and R. Ziff, Chem. Eng. Sci. 44:1403 (1989). 